\title{
Percepciones de los empresarios de Pymes rurales sobre la integración de las TIC
}

\author{
Mª Cruz Sánchez Gómez 1 , Beatriz Palacios Vicario ², Camino López García ${ }^{1}$, Ana \\ Sánchez García ${ }^{1}$ \\ mcsago@usal.es, camino_lopez@usal.es, bpalaciosvi@upsa.es, asg@usal.es \\ ${ }^{1}$ Facultad de Educación, Universidad de Salamanca, 37008, Salamanca, España \\ ${ }_{2}^{2}$ Facultad de Psicología, Universidad Pontificia de Salamanca, 37002, Salamanca, España
}

DOI: 10.17013/risti.e2.71-84

\begin{abstract}
Resumen: El estudio es el fruto de una iniciativa cuya finalidad fue conocer las percepciones que poseen los empresarios de las pymes del ámbito rural con respecto a la utilización de las TIC en su actividad laboral. El objetivo principal es proporcionar una visión contextualizada de la utilización de las herramientas tecnológicas por parte de este sector empresarial, para identificar necesidades e implementar programas de formación, actualización y mejora profesionales del sector. El Centro Internacional de Tecnologías Avanzadas (CITA), ha puesto en marcha este proyecto que pretende obtener datos que faciliten crear una oferta formativa ad hoc. La metodología es de carácter cualitativo, recogiendo información de grupos focales en los que han participado 48 empresarios. Los resultados reflejan falta de información sobre el uso y potencialidades de las TIC necesitan asesoramiento para optimizar recursos, conseguir personal técnico y cursos de formación adaptados a las demandas.
\end{abstract}

Palabras-clave: percepciones, actitudes, creencias, pequenas empresas, investigación.

\section{Perceptions of entrepreneurs in rural SMEs in relation to ICT integration}

Abstract: This study is the result of an initiative aimed at learning the perceptions that owners of SMEs in the rural context about the use of ICT in their work. Our main objective is to provide a practical view of the use of technological tools in the business sector in order to identify specific needs and implement programmes for training, updating, and improvement addressed to both entrepreneurs and their employees. The Internacional Center of Advance Technologies (CITA) has put this programme into practice with a view to obtaining the information necessary to create ad hoc training programmes. A quantitative methodology is used, and data were collected through focal groups in which participated 48 employers. The results reflect a lack of information on the use and potential of ICTs need advice to optimize resources, ensure technical and training courses tailored to the demands staff. 
Keywords: perceptions; attitudes; beliefs; small businesses; research.

\section{Introducción}

En el contexto de crisis actual los negocios tienden a renovarse para poder ofrecer nuevas ofertas con recursos tecnológicos ofrecidos por las Tecnologías de la Información y del Conocimiento (TIC) en las empresas. Eficiencia, ahorro, inmediatez. son algunos de los calificativos que caracterizan a las nuevas tecnologías, pero aún con esta reputación podemos observar una resistencia a su aceptación por parte de los pequeños y medianos empresarios.

Las Pymes constituen los agentes dinamizadores y estabilizadores de la ciudadanía. Son varios los condiconantes del desarrollo de estas empresas que aportan un retraso si las comparamos con la actividad que se desarrolla en las ciudades. Esto tiene un efecto directo sobre la integración de las TIC en el contexto empresarial, alimentando la brecha digital. Esta teoría es respaldada por las últimas investigaciones en este contexto (Subdirección General de Apoyo a la Pyme, Dirección General de Industria y de la PYME, Ministerio de Industria, Energía y Turismo, Gobierno de España, 2013; SAGE, 2013; Observatorio Nacional de la Telecomunicación y la sociedad de la información, 2013). El uso de herramientas tecnológicas ayudan a poner en marcha, potenciar y expandir las empresas y se han consolidado como un sector clave para el desarrollo económico, especialmente en el momento económico negativo como el actual, en el que constituyen el foco de nuevas percepciones, creencias y conductas en los ciudadanos ofreciendo nuevos desafíos para las empresas. Se configuran como elementos clave para mejorar la competitividad de un país, las exportaciones, y conseguir crear nuevos negocios y empleo (Fundación Telefónica, 2012)

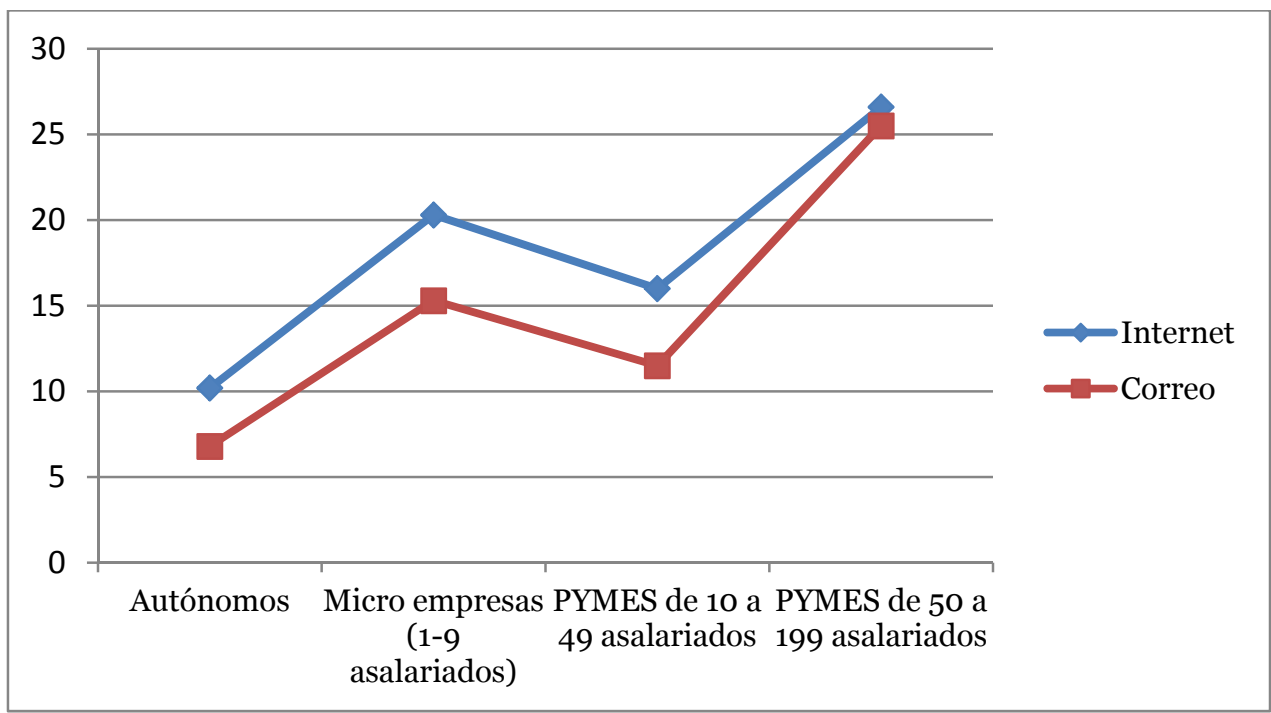

Figura 1- Disponibilidad de servicios de Internet y correo (España) (Tomado del Informe Telefónica elaborado por la Fundación Telefónica, 2010) 
Está comprobado que nivel de utilización de las TIC en las empresas guarda una relación directamente proporcional el tamaño de las mismas, "ya que las más pequeñas suelen tener más restricciones presupuestarias a la hora de abordar inversiones cuyos beneficios se obtienen generalmente a medio plazo". (Fundación Telefónica, 2012, p. 68).

En 2010 el 84,8\% de las empresas PYMES entre 50 y 199 asalariados tienen su propia página web aunque solo un 16,6\% realiza comercio electrónico (Informe Telefónica, 2010):

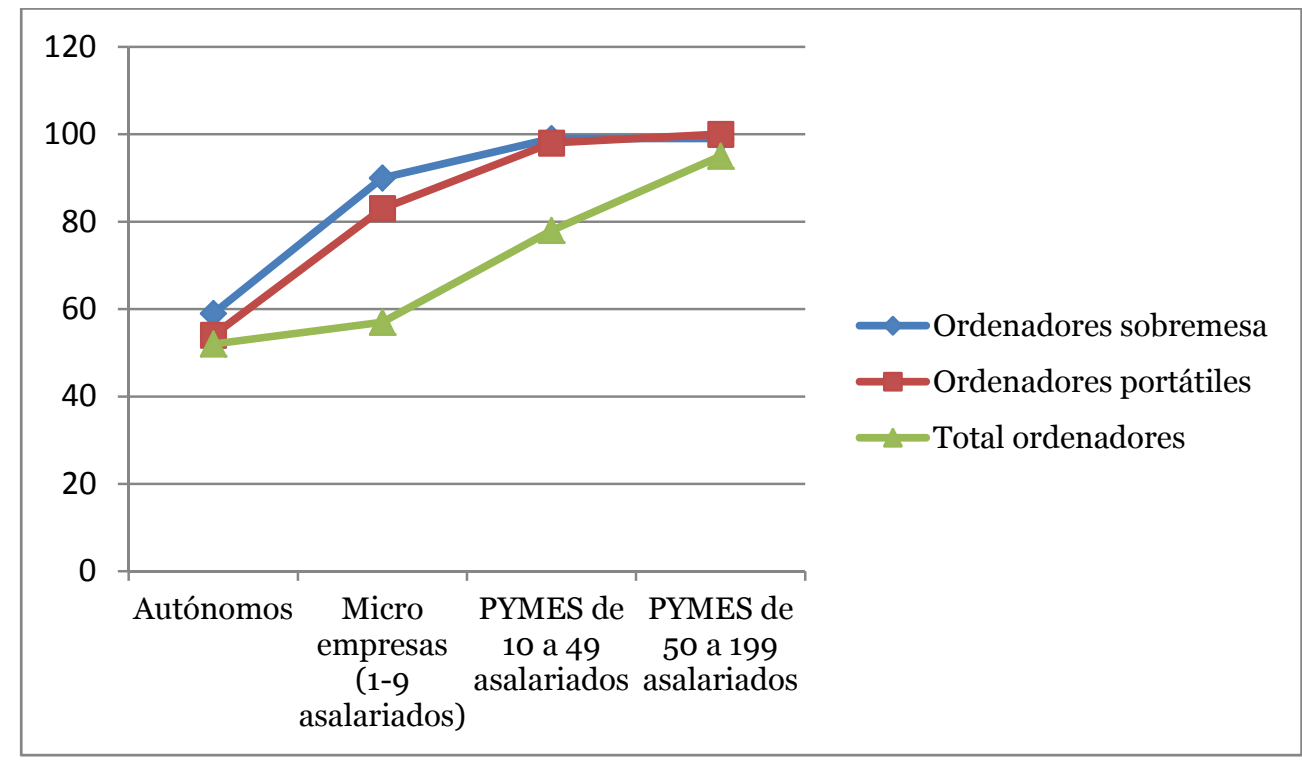

Figura 2 - Disponibilidad de ordenadores en PYMES, (Tomado del Informe Telefónica elaborado por la Fundación Telefónica, 2010)

Por esta razón el Centro Internacional de Tecnologías Avanzadas, CITA, ha puesto en marcha el proyecto Timepyme en la comarca de Peñaranda de Bracamonte (Salamanca). Dicho proyecto se centra en el análisis en las PYMEs porque constituyen el mayor porcentaje de las empresas en España. Es la fase local del programa marco llevado a cabo a nivel nacional y regional (Programa Operativo de Cooperación Transfronteriza España-Portugal 2007-2013, y el Programa Operativo de Cooperación Transfronteriza España-Portugal 2007-2013, Provincia de Salamanca). Su principal objetivo es integrar las TIC en el tejido empresarial de las pymes de la zona rural para mejorar su productividad.

El trabajo de investigación ha sido realizado por un equipo de investigación integrado por personal del CITA y de la Universidad de Salamanca, y tiene como finalidad recopilar información, analizarla y orientar sus resultados hacia la creación de un Modelo de Capacitación Tecnológica para Pyme. Los resultados que se presentan permiten conocer la realidad de las Pymes y las microPymes en la comarca de 
Peñaranda de Bracamonte (Salamanca) derivada del impacto de las TIC en su actividad empresarial.

Las conclusiones advierten de una integración tardía y ritmo lento de las TIC en las pequeñas empresas españolas. Parece que el volumen de recursos TIC en las empresas está relacionado proporcionalmente con el número de trabajadores que la integran.

\section{La investigación}

\subsection{Objetivo}

Este trabajo de investigación tiene por objetivo conocer las percepciones de los empresarios de la comarca de Peñaranda de Bracamonte, Salamanca, hacia la integración de las TIC en el contexto de las Pymes y Micropymes.

Con los resultados pretendemos aportar una visión real del actual estado de integración de las TIC en el tejido empresarial, así como conocer las necesidades y demandas de los empresarios. Esperamos promover acciones que den solución a las carencias detectadas como la creación de tiendas virtuales y marketing online.

\subsection{Descripción de la metodología}

La metodología que se ha adoptado es de carácter cualitativo, recogiendo datos a través de los grupos focales en los que han participado empresarios de la zona de Peñaranda de Bracamonte. En situaciones como las planteadas en este estudio, en que los contextos a investigar están claramente definidos, un planteamiento meramente extensivo no permitiría la realización de un análisis minucioso como el que propone el enfoque intensivo que nos ocupa (Sánchez, 2006; Denzin, \& Lincoln ,2011).

Por estas razones teórico-metodológicas y pragmáticas, se considera pertinente la utilización de métodos cualitativos para identificar y proponer líneas de intervención ad hoc sobre la incorporación de las TIC en el tejido empresarial.

Previo al análisis de los datos se efectuaron las transcripciones del los grupos entrevistados (Sánchez \& Revuelta, 2005). A continuación el grupo de investigación efectuó la lectura comprensiva de los textos transcritos para identificar la ideas relevantes maifestadas por los empresarios. Tras este momento, se constituyeron las categorías o dimensiones que nos permitieron analizar los datos (categorización). Teniendo en cuenta el contenido de los discursos producidos en las sesiones y los estudios teóricos sobre las dimensiones identificadas, se establecieron 7 macrocategorías de análisis con sus correspondientes subcategorías. El resultado fue el siguiente mapa de categorías, ordenadas de forma jerárquica, que muestra las líneas discursivas de los grupos de empresarios: 


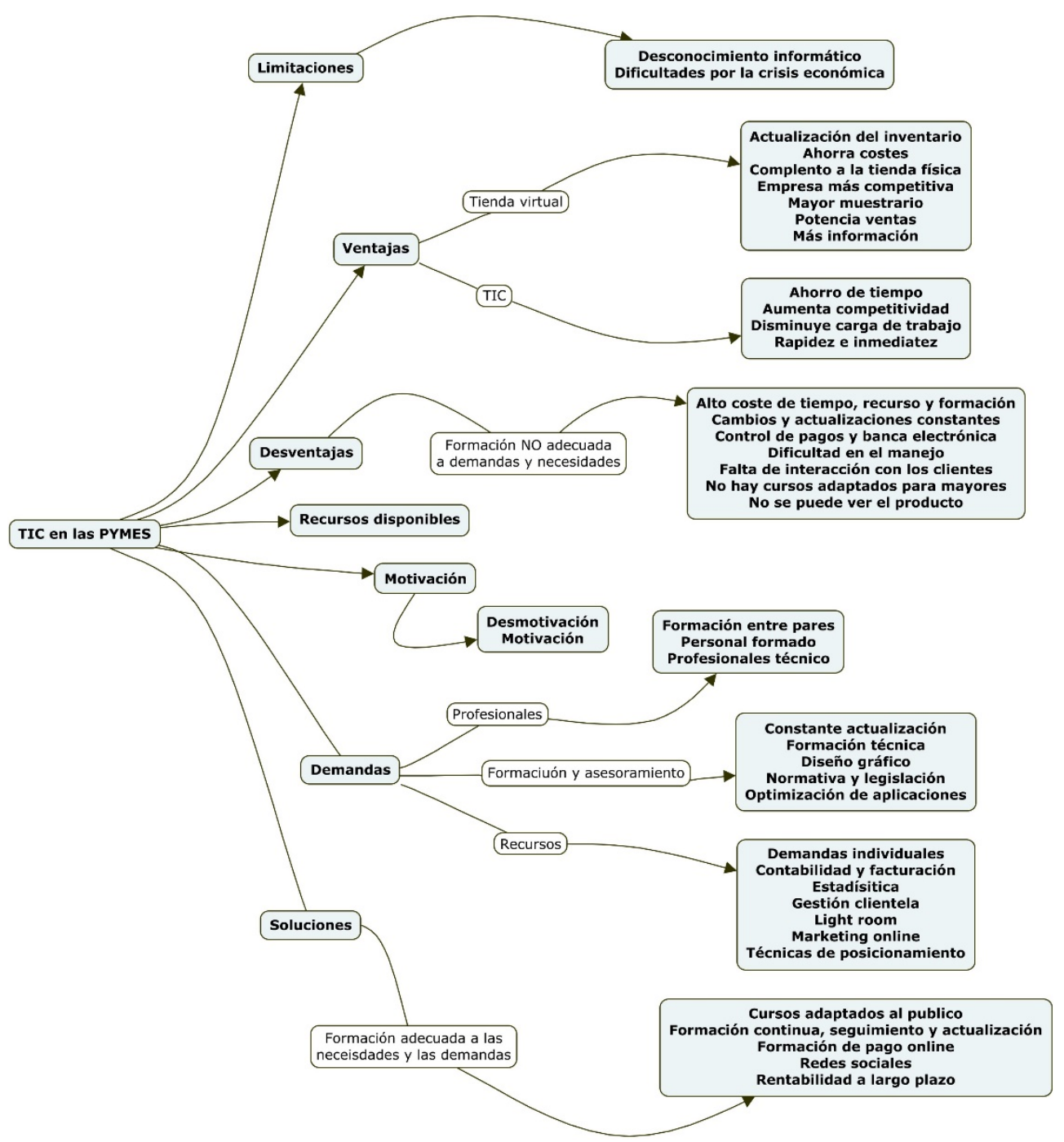

Figura 3 - Mapa de categorías. Elaboración propia.

Durante el estudio se han tenido en cuenta además una serie de variables recogidas a través de un cuestionario elaborado ad hoc que se especifican en el siguiente apartado y que han servido para conocer las características contextuales y muestrales. Dicho cuestionario fue cumplimentado por los empresarios que participaron en los grupos de discusión.

\subsection{Variables}

Las variables que se presentan a continuación están ampliamente relacionadas con los empresarios, por un lado, y las empresas y el contexto por otro. Estas variables son:

a) Sociodemográficas (datos sobre la empresa y el empresario) 

b) Técnico informáticas
c) De aplicación a la gestión emprearial
d) Mantenimiento y seguridad
e) Normativas

\subsection{Trabajo de campo}

Se desarrolló entre los meses de febrero a mayo de 2013 en el contexto del CITA, en las instalaciones que el centro posee en Peñaranda de Bracamonte, Salamanca.

Se realizaron cinco grupos de discusión con un total de 48 participantes. Esta muestra fue sufienciente para llegar a la saturación del discurso, es decir, la no presencia de elementos nuevos en el discurso (Sánchez , 2008b; Sánchez, Delgado \& Santos, 2013). Dichos grupos fueron moderados por miembros del grupo de investigación.

\subsection{Descripción de la muestra.}

La muestra se ha constituido por muestro teórico sin establecer ningún tipo de clasificación más allá de que cumpliesen con el requisito de ser empresarios responsables de Pymes y/o microPymes en la comarca de Peñaranda de Bracamonte (Salamanca) factor imprescindible para participar en este estudio. Sus características son:

\section{a) Sociodemográficas (datos sobre la empresa y el empresario)}

Los sujetos tienen edades comprendidas entre los 23 años y los 75 años, siendo la media 42 años. De los 48 empresarios, 38 son hombres y 10 mujeres (79 y $21 \%$ respectivamente) adaptándose este datos a la realidad sociodemográfica empresarial de la comarca. El 48 \% de los empresarios ha cursado estudios medios, el 35\% superiores y un $17 \%$ estudios básicos.

El número de años trabajados oscila entre 2 y 50 años de experiencia laboral, situándose la media en 15 años. Las empresas tienen una media de 6 trabajadores, y la mayoría son del sector secundario. Los empresarios proceden en su mayor parte de la comarca de Peñaranda, pero también hay de otros lugares de España y Europa: Ávila, Cáceres, Bilbao, Madrid, Salamanca, San Sebastián, Alemania y Australia. El número de trabajadores oscila entre 1 y 40.

En cuanto al volumen de actividad empresarial: el 21,4 \% de las empresas factura entre 600.000 y 1,5 mil euros anuales; $14,3 \%$ factura entre 100.000 y 300.00 euros anuales; un 9,5\% de 2 a 10 mil; el 7,1\% menos de 100 mil; y, por último, el 2,4\% factura entre 1,5 a 2 mil euros anuales. Solo el 16,7 \% de los empresarios ha confirmado que dentro de su presupuesto existe una partida dedicada a la inversión en Tecnología de la Información y Comunicación (TIC).

\section{b) Técnico informáticas}

En el $100 \%$ de las empresas se dispone de ordenadores. El 60,4 \% afirma que sus computadoras tienen una antigüedad entre 1 y 3 años, frente a un 35,4 \% de tres años y 4,2 \% con menos de un año. Respecto a los dispositivos periféricos, en el $92 \%$ disponen de impresoras; un 85 \% usa escáner; 19 \% utilizan lectores de códigos de barras, mientras que el $15 \%$ usa otros dispositivos. Windows es el sistema operativo más utilizado. A los servidores se destina: Acceso a Internet (37\%); copias de seguridad (29\%); el 27\% al uso compartido de carpetas y el 7\% a los distintos dominios. 
Todos disponen de conexión a Internet, siendo la conexión ADSL la más utilizada, con un 75\%. Un 13 \% hace uso de 3G. Solo un 8\% usa la Red Telefónica Básica/Red Digital de Servicios Integrados - RTB/RDSI -, mientras que el $2 \%$ accede a través de Cable Satelital y otros.

En mayor proporción en las empresas de los empresarios entrevistados se usa Internet para acceder a la banca electrónica (60\%), luego, prácticamente en igual medida, para gestionar pedidos (54\%), adquirir productos (56\%), gestionar asuntos relacionados a la administración pública (52\%) y buscar innovaciones y novedades (52\%). Se le da menor uso para comunicar informaciones a los empleados (15\%) u organizar y planificar el equipo comercial (15\%).

\section{c) Aplicación a la gestión empresarial}

Las aplicaciones informáticas se usan principalmente para gestionar la contabilidad de la empresa (36\%), los sistemas informáticos (32\%) y el control de los almacenes (21\%).

El mayor porcentaje es el dedicado a la facturación (37\%), seguido de la gestión de pedidos (26\%). El apartado con menor cantidad de funcionalidades específicas es la gestión de nóminas (6\%).

\section{d) Sistema de trabajo colaborativo}

Las herramientas de trabajo colaborativo especificadas son las bases de datos, carpetas compartidas y portales Web, se les da prácticamente el mismo grado de utilización (19, 19 y $16 \%$ respectivamente).

El 57 \% de las empresas utiliza el correo electrónico para comunicarse con el exterior, dígase, otras empresas, clientes, proveedores y demás.

El $51 \%$ de las empresas tiene presencia en Internet a través de sus propias páginas Web. Un $24 \%$ por medio a portales relacionados al sector en el cual se desenvuelven, y en menor medida tiene presencia a través de tiendas virtuales (9\%), blogs y otros (7\%) y, finalmente, el medio menos utilizado es el E-Marketing, utilizándolo solo el 2\% de los empresarios encuestados.

Cabe destacar que aunque tienen más presencia en Internet a través de la Web, este no es el medio más utilizado para contactar con el exterior

A diferencia de los resultados presentados en los estudios anteriores, donde casi el 100\% utilizada estos servicios para mostrar información sobre la empresa, en este caso se utiliza más para consultas (28\%) y para presentar su catálogo de productos (24\%). La información de la empresa queda en un tercer lugar con un $21 \%$.

Los miembros de la compañía que por alguna razón deben desplazarse fuera de esta utilizan en mayor proporción los sistemas de localización (100\%), aplicaciones SFA/FFA y la red interna (98\% c/u). En menor media el teléfono (61\%), el correo/web (36\%). El resto de vías de comunicación son usadas entre un 4 y $9 \%$.

Esto muestra un cambio de patrones, ya que en el estudio anterior el medio más utilizado era el teléfono, con un $100 \%$ de uso.

Asimismo, aproximadamente el 50\% de las empresas dispone de dispositivos informáticos portátiles, como las PDA y los PC Portátiles. Disponen de tabletas solo en 
un 30\% de los casos, mientras que el uso de los dispositivos industriales y otros, son usados en no más de un 10\%.

e) Mantenimiento y seguridad

El $65 \%$ de las empresas dispone de algún sistema de mantenimiento y seguridad, centrados principalmente en el uso de copias de seguridad locales (31\%) y en el uso de servidores (31\%).

Respecto a la utilización de algún sistema de autenticación digital, el $62 \%$ no posee pero el restante $38 \%$ sí, destaca el hecho de que están muy enfocados a la tramitación administrativa (50\% respecto al resto de opciones).

\section{f) Normativas.}

El 38\% conoce la obligatoriedad de declarar los ficheros sobre datos y el 24\% cuenta con asesoría al respecto. Mientras que solo el 19\% de ellos dispone de aviso legal o conoce el marco legal que regula el uso de las TIC.

\section{Resultados}

Mediante análisis de contenido y con ayuda del software Nvivo 10 (Palacios, Gutiérrez \& Sánchez, 2013) se obtuvieron resultados que hacen referencia al porcentaje de discurso analizado correspondiente a cada una de las categorías propuestas en el mapa conceptual (Figura 3). Durante esta fase se garantizaron los criterios de calidad en investigación cualitativa (Lincoln y Guba, 1985; Guba y Lincoln, 1989; Sánchez, Delgado y Santos, 2013, Palacios, Gutiérrez \& Sánchez, 2013) que quedan sintetizados como sigue: la codificación se realizó por varios miembros del equipo de investigación para así poder garantizar la credibilidad (validez interna) del proceso. Para probar la dependencia (fiabilidad) del sistema de categorías y garantizar la homogeneidad y coherencia en la codificación se elaboró un manual descriptivo que se puso a disposición de los codificadores expertos en el tema. Por último, la objetividad (Confirmabilidad) se ve reflejada mediante la aportación de fragmentos literales del discurso de los empresarios.

A continuación se describen los resultados del análisis de contenido de las opiniones que los empresarios manifiestan sobre las TIC en el contexto de las pequeñas empresas (PYMES y microPYMES).

El tema principal que vertebró el resto de temáticas abordadas fue el uso de las TIC. Con todo ello se fue creando un mapa del discurso que nos mostró las percepciones hacia las TIC y el mundo de la empresa. 


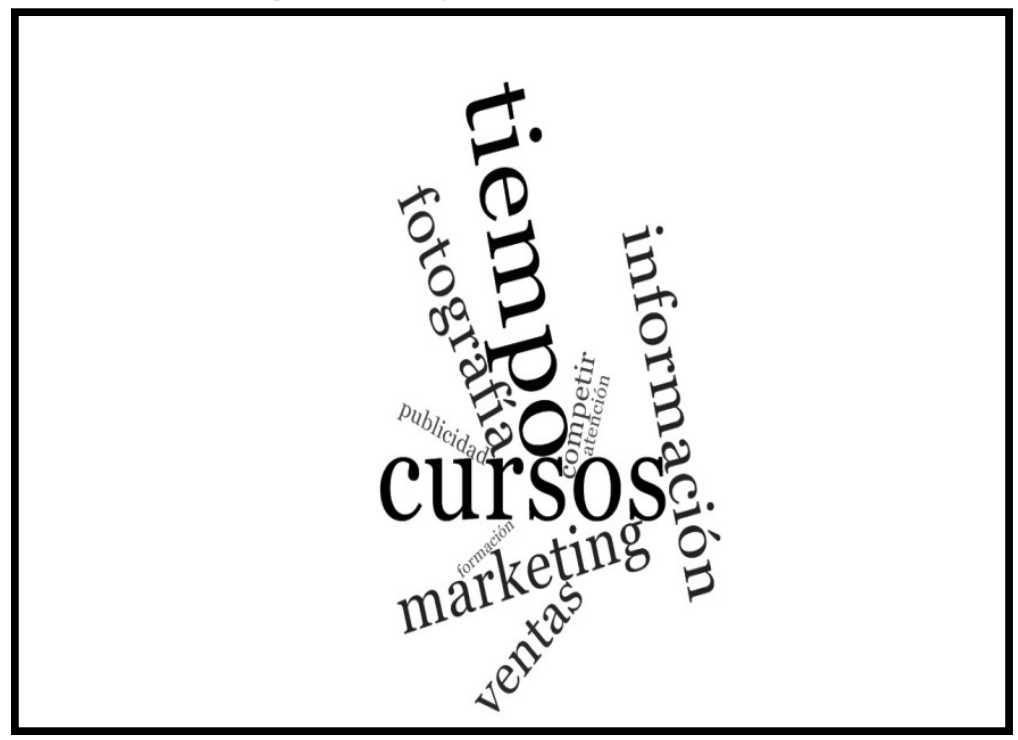

Figura 4 - Elementos clave del discurso acerca de las TIC en la empresa. Elaboración propia con el software de análisis cualitativo NVIVO10

En la figura 4, se perciben las diferentes categorías que aparecen en los grupos focales. Se observa que a nivel general se dedica una gran parte del discurso a hablar sobre las ventajas, desventajas de las TIC y de las tienda virtuales, de las demandas, la motivación o desmotivación que les produce enfrentarse a las TIC, la creatividad, las limitaciones y las redes sociales. Y también dedican una importante parte del tiempo de discusión a hablar sobre sus conocimientos y la gran cantidad de tiempo en formación sobre nuevas tecnologías que es necesario.

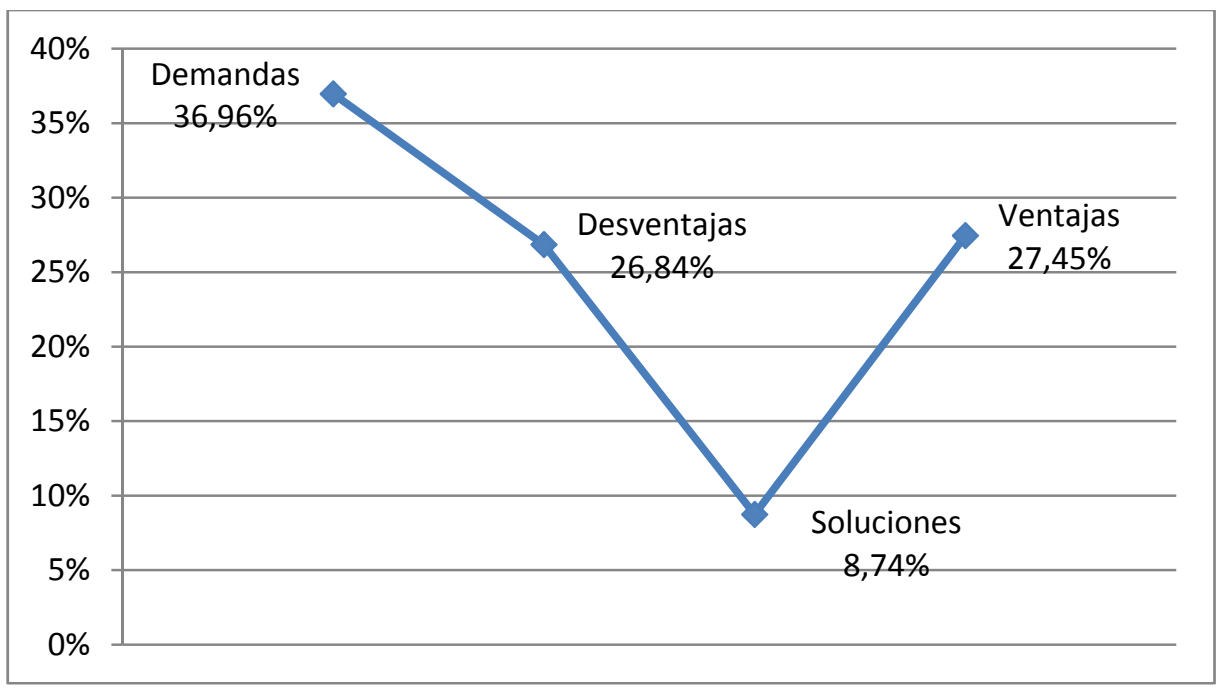

Figura 5 - Porcentaje de referencias codificadas en las principales categorías. Elaboración propia 
La percepción de los empresarios sobre las TIC es la "ayuda” que estas herramientas les pueden prestar en sus actividades empresariales y son conscientes de la necesidad de su utilización para dinamizar, en estos tiempos de crisis, sus negocios. Al respecto, los empresarios manifestaban estas cuestiones de la siguiente forma:

"Internet es un escaparate dal mundo, es está comprobadísimo, que lo habjábamos ayer"

"Yo solamente a la hora de hacer pedidos manejando como ha dicho Álvaro el correo electrónico, que dices bueno es mucho más rápido o tienes que hacer una pregunta y antes cogías el teléfono o hay días que coges y mandas el correo, haces la foto, la metes, te contestan, o sea en ese tema si pero en otro tema no."

Estas creencias y actitudes se basan en gran medida en lo que estos empresarios consideran como ventajas e inconvenientes determinadas acciones tecnológicas.

La mayoría de los empresarios mantienen un discurso positivo hacia las TIC, destacando como principal ventaja la rapidez para sus tareas diarias, así como la inmediatez para determinadas funciones que antes requerían de un tiempo para su tramitación y el ahorro de costes, elemento principal en estos tiempos de crisis.

"Si, de hecho nosotros hemos pasado de recibir miles de fax a solo y exclusivamente utilizar un email, o incluso si llega algún fax está conectado al ordenador para no tener que imprimir en papel."

"la factura. Otro tema, que lo tiene Lotus, vienen y le digo pico y le digo, dime las facturas del uno al quince y me dice cinco facturas, pues quiero esta, plaf picas el botón, ya está metido todo el género y dices esto es una maravilla”.

La principal desventaja es la falta de formación y en concreto acerca del control de pagos y banca electrónica. Otra desventaja que ven es la inadecuación de recursos a las necesidades de la empresa, así como la imposibilidad de ver el producto tal cual es, ya que las imágenes en ocasiones dependen de la resolución del ordenador en el que se ven. También destacamos como inconvenientes la falta de cursos para empresarios de más edad, el escaso conocimiento en el manejo de herramientas y sus posibilidades. Por otra parte el continuo cambio en las nuevas tecnologías y el alto coste, en tiempo, recursos y formación inicial también es un hándicap a considerar. Algunos de sus discursos al respecto son los siguientes:

"Yo también por casualidad pero ya cuando vosotros no habíais nacido, entre casi en informática, y claro, lo más esencial y claro aquello parecía chino, ayer te lo dije a ti, me parece que me están hablando en chino, porque había muchas partes técnicas que no se utilizan, como persona que soy yo en internet”.

"El trato con el cliente a través de la tienda virtual creo que no es tan cercano como el que puedes llegar con la forma directa."

"Lo que aprendes un día al día siguiente te lo actualizan" 


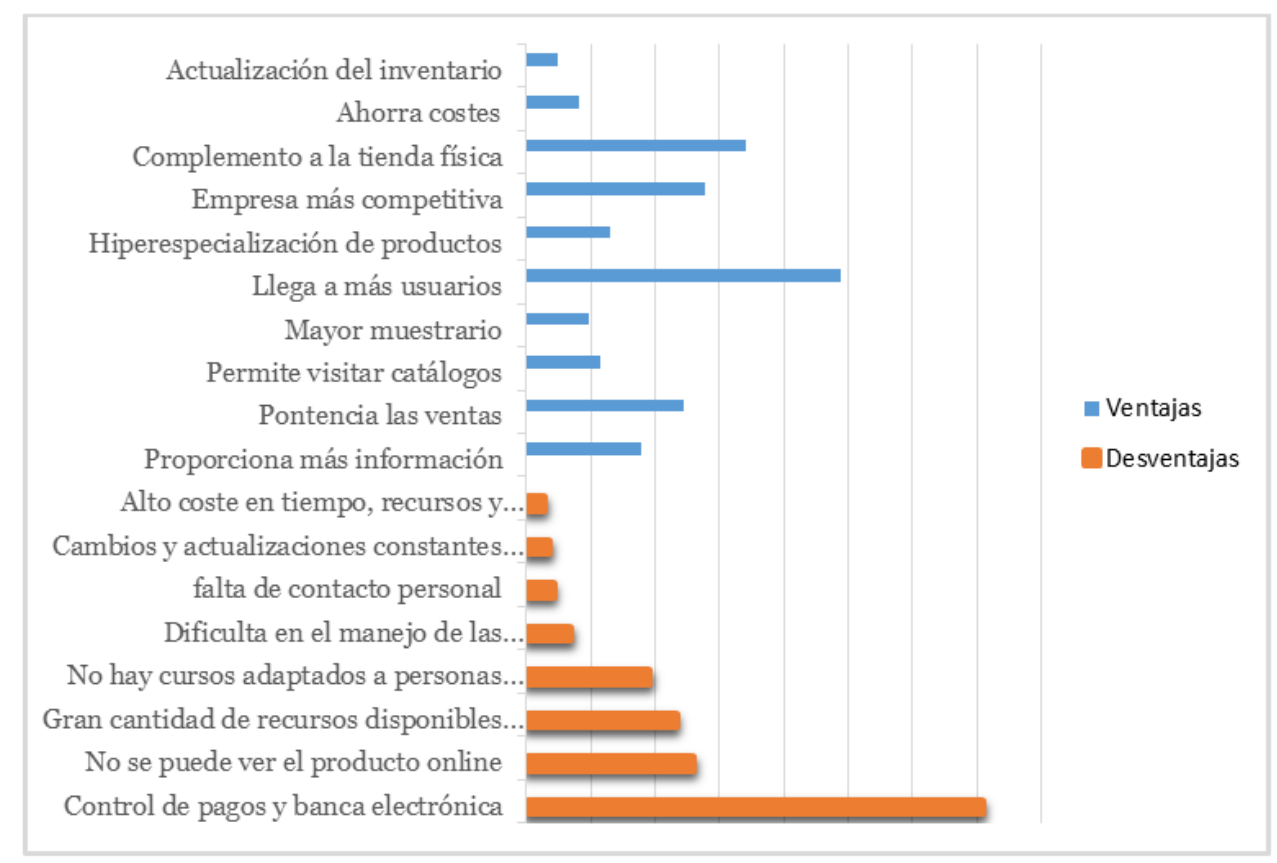

Figura 6 - Ventajas y desventajas de las TIC en la empresa. Elaboración propia

Teniendo en cuenta estas ventajas y desventajas es necesario conocer y analizar las demandas que realizan los sujetos y que son coherentes a las necesidades que presentan. Lo que más demandan los empresarios es la formación, después los recursos y a continuación los profesionales adecuados.

En relación con las demandas formativas, hay una serie de ellas más consolidadas entre los empresarios y que están relacionadas con la necesidad de formación permanente, formación técnica, conocimientos en fotografía y diseño, conocimiento de la legislación relacionada con el uso de las TIC a nivel empresarial y también información acerca de la optimización de las aplicaciones. Éstas están muy vinculadas al hecho de que algunos de estos empresarios están en fase de formación para la puesta en marcha de una tienda virtual en sus negocios y sus demandas giran en torno a esa preocupación

Destaca también la demanda de recursos como el Marketing online que resolvería cuestiones relacionadas con la venta del producto a través de la imagen que puede verse distorsionada. Reclaman también el conocimiento de técnicas de posicionamiento, ya que en los grupos de discusión hablaban de ciertos mecanismos para que determinadas páginas saliesen siempre en los primeros resultados de una búsqueda en los distintos navegadores. La contabilidad y facturación es una demanda que hacen en relación a programas informáticos que ayudan a llevar la contabilidad de la empresa, los cuales simplemente a través de la lectura del código de barraras de un producto, este queda registrado en el sistema. La gestión de la clientela es también un 
recurso demandado por los empresarios, la cual les ayudaría a conocer la demanda y ajustar la oferta. Y en menor medida, demandan cursos de diseño gráfico, cómo hacer estadísticas, conocer otros modelos de tienda virtual para diseñar la propia, y programas específicos como el PHOTOSHOP para la mejora de la venta en relación a la exposición del producto.

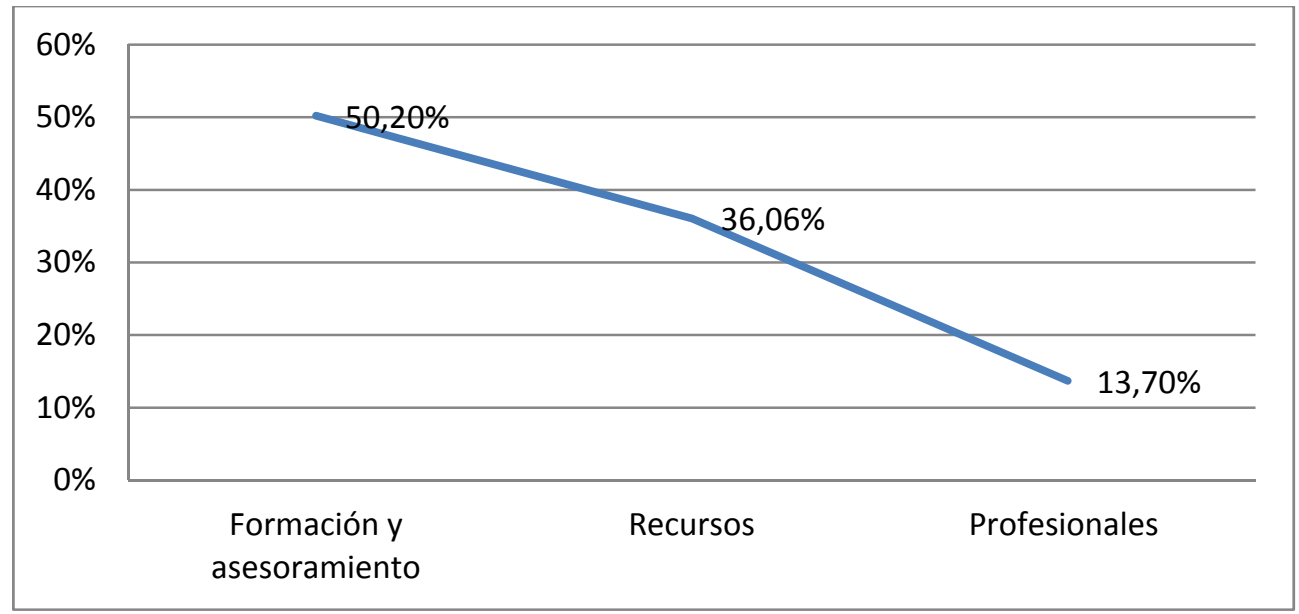

Figura 7 - Principales demandas relacionadas con las TIC y la empresa. Elaboración propia.

"Una cosa en montar una tienda virtual y otra cosa es tener conocimientos de marketing y todas estas historias".

"Yo creo que aparte de intentar cubrir las bajas en ventas que todo el mundo tiene, es que hoy en día si una empresa no está en internet, o buscar en internet y no aparece tu empresa es como si no asistiera, entonces simplemente que aparezca, busques juguetería en Peñaranda, te aparece tu tienda y no aparece el Corte Inglés de Salamanca, sabes, entonces alguien ya sabe que existes y que estas aquí."

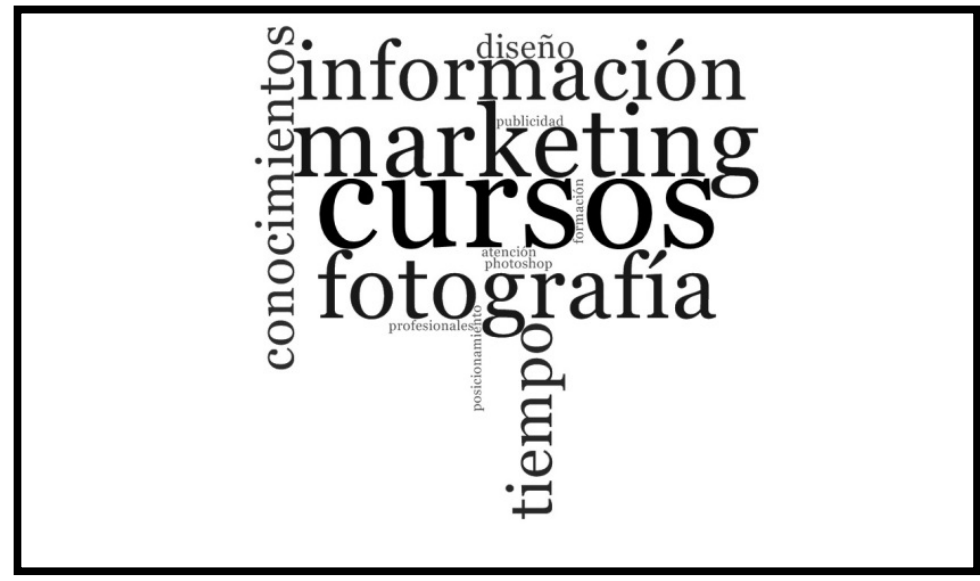

Figura 8 - Principales demandas relacionadas con Formción y asesoramiento. Elaboración propia con el software de análisis cualitativo NVIVO10 
Finalmente, consideramos que teniendo en cuenta estas demandas, se pueden establecer líneas de actuación para los empresarios de la comarca, de manera que estos adquieran los conocimientos, habilidades y tecnicas que les lleven a tener presencia en la red dinamizando así el tejido empresarial de la comarca. Estas actuaciones deberán conjugar los distintos tipos de necesidades para prestar una ayuda más específica y cualificada sobre la implementación y utilidad de las nuevas tecnologías en la pequeña y mediana empresa.

\section{Conclusiones}

Los empresarios de la comarca de Peñaranda de Bracamonte conocen, tienen recursos relacionados con las Nuevas Tecnologías y son conscientes de sus posibilidades. Están sensibilizados acerca de la necesidasd del uso en sus negocios pero sienten ciertos temores relacionados con el desconocimiento de ciertas cuestiones de aplicación y optimización de las mismas, dado el elevado coste de alguna de ellas. Sus demandas están relacionadas directamente con la mejora del uso que ya hacen, la mayoría de empresarios, de ciertas aplicaciones en sus empresas. Consideramos que dichas demandas han de materializarse en acciones en torno a tres ejes fundamentales: formación, recursos y profesionales de apoyo. Actualmente teniendo en cuenta estas conclusiones, el CITA está llevando a cabo un primer ciclo de cursos relacionados con aspectos técnicos y legislativos necesarios para implantar una tienda online, y para el cuarto trimestre del año pondrá a disposición de los empresarios un segundo ciclo de cursos más especificos para software sobre comercio y marketing online. En el primer ciclo de cursos están matriculados los mismos empresarios que han participado en este estudio, y exiten tres tiendas virtuales en proceso de montaje.

\section{Agradecimientos}

Agradecemos al CITA la disponibilidad de medios y recursos materiales como humanos, para que esta investigación se hiciese posible, y a los empresarios de las pequeñas y medianas empresesa de la Comarca de Peñaranda que desinteresadamente han colaborado como muestra del estudio.

\section{Referencias}

Denzin, N.K., \& Lincoln, Y.S. (2011). Handbook of Qualitative Research. Londres: Sage

Guba, E. \& Lincoln, Y. (1989). Fourth generation evaluation. Newbury Park: Sage.

Fundación Telefónica (2010). La sociedad de la Información en España en 2010. Disponible

en http://www.fundacion.telefonica.com/es/arte_cultura/publicaciones/sie/sie2010. htm

Fundación Telefónica (2012) La sociedad de la Información en España en 2012. Disponible http://www.fundacion.telefonica.com/es/arte_cultura/publicaciones/sie/sie2012. htm 
Lincoln, Y.S. \& Guba, E.G. (1985). Naturalistic inquiry. Beverly Hills: Sage.

Observatorio Nacional de la Telecomunicación y la sociedad de la información (ONTSI) (2013). La sociedad en Red, informe anual 2012. Las TIC en las Pymes y Grandes empresas españolas.

Palacios, B.; Gutiérrez, A. \& Sánchez, M.C. (2013). NVIVO 10: Una herramienta para la investigación en Comunicación. In Pacheco, M. Vicente, M. y González, T. (coords) Investigar la Comunicación hoy. Revisión de políticas científicas y aportaciones metodológicas: Simposio Internacional sobre Política Científica en Comunicación, (4), 1003-1018

SAGE. (2013) Radiografía de la Pyme 2013. Disponible en http://www.sage.es/recursos-de-negocio/observatorio-sage/radiografia-de-lapyme-2013

Sánchez, M.C. (2006). Estrategias de investigación en Ciencias Sociales. Comunicación y Periodismo, 2 , 9-37.

Sánchez, M.C. \& Revuelta, F. I. (2005). El proceso de transcripción en el marco de la metodología de investigación cualitativa. Enseñanza-anuario interuniversitario de didáctica, 23, 367-386.

Sánchez, M.C. (2008b). La calidad en la investigación cualitativa. Investigación y tecnologías de la información y comunicación al servicio de la innovación educativa, 241-265. Salamanca: Estudios Científicos USAL.

Sanchéz, M.C.; Delgado; M.C. \& Santos, M.C. (2013). El proceso de la Investigación cualitativa. Manual de procedimiento: ejemplificación con una tesis doctoral. Valladolid: Edintras

Subdirección General de Apoyo a la Pyme, Dirección General de Industria y de la PYME, Ministerio de Industria, Energía y Turismo, Gobierno de España (2013). Retrato de las Pymes 2013. 